\title{
Alitretinoin - schwere Handekzeme heilen ab
}

- Toctino ${ }^{\circledR}$ (Alitretinoin) vom Unternehmen Basilea Pharmaceutica ist das erste und einzige zugelassene Medikament gegen schwere chronische Handekzeme, die nicht auf starke topische Kortikoide ansprechen. Mit seinem Wirkstoff Alitretinoin ist es das einzige Präparat, das speziell für die Indikation „Handekzem“ zugelassen ist. Ein effektives Medikament ist für diese Patientengruppe deshalb so wichtig, weil die entzündliche Hauterkrankung als häufigste Berufsdermatose gilt.

Die Wirksamkeit des Vitamin-A-Derivats ist seit Längerem auch im Zuge von Phase-IIIStudien belegt [siehe etwa Br J Dermatology 2008; 158: 808]. Sie zeigten, dass unter einer Dosierung von $30 \mathrm{mg}$ einmal täglich die Läsionen bei 48 Prozent vollständig oder fast vollständig zurückgehen, unter $10 \mathrm{mg}$ bei 28 Prozent, unter Placebo hingegen nur bei 17
Prozent. Zudem setzt die Wirkung der Therapie rasch ein: Bereits in der Frühphase bessern sich Rötung, Juckreiz, Bläschen, Entzündung, Ödeme und die Schmerzen durch Hautrisse. Eine Verschlechterung ist nach dem Absetzen nicht zu befürchten, eine ein- oder ausschleichende Dosierung nicht nötig.

Die Deutsche Dermatologische Gesellschaft hat den Wirkstoff bereits kurz nach der Zulassung in ihre Leitlinie zum Management von Handekzemen aufgenommen [JDDG 2009; 7 (Suppl. 3): 1]. Seit Kurzem empfehlen ihn auch die italienischen Leitlinien.

Seit der Markteinführung in mehreren europäischen Ländern - in Deutschland im November 2008-wurden bereits mehr als 8.000 Patienten mit dem Vitamin-A-Derivat behandelt. Sie nehmen die Kapseln in Dosierungen von $10 \mathrm{mg}$ oder von $30 \mathrm{mg}$

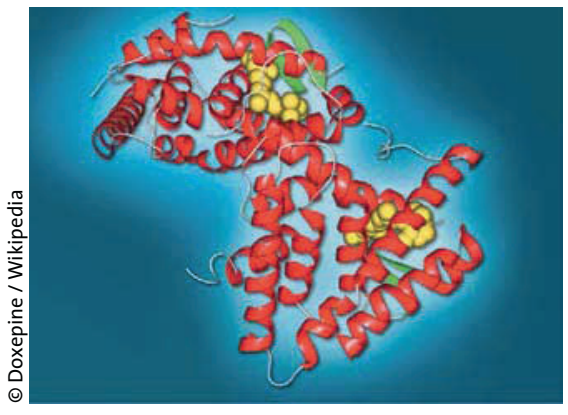

Toctino $^{\circledR}$ (Alitretinoin) lagert sich wie hier Retinoinsäure an den Rezeptor RXR.

einmal täglich zu den Mahlzeiten. Patienten, deren Ekzeme sich in den ersten 12 bis 24 Wochen nicht hinreichend bessern, profitieren von einer Einnahme über weitere 12 bis 24 Wochen. Das Präparat ist auch auf Dauer gut verträglich, mit der fortgesetzten Einnahme sind keine zusätzlichen unerwünschten Wirkungen verknüpft. ars

Nach Informationen von

Basilea Pharmaceutica

\section{Ustekinumab gegen die Plaque-Psoriasis}

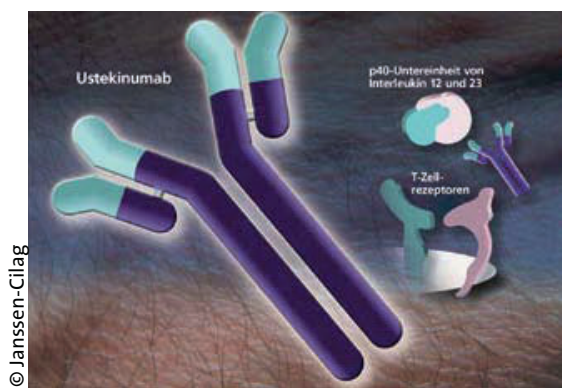

Ustekinumab bindet an die p40-Untereinheit, einen Bestandteil der Interleukine 12 und 23. Dadurch wird der Entzündungsprozess spezifisch gestoppt und die übermäßige Produktion von Hautzellen normalisiert.
Blockade der pathologischen Reaktionen zugeschnitten. Was auf wissenschaftlicher Grundlage entworfen worden war, bewährte sich in der klinischen Praxis. Das belegen die Zulassungsstudien PHOENIX 1 und 2, publiziert im Mai 2008 [Lancet 2008; 371: 1665; 1675).

Die knapp 2.000 Teilnehmer mit mittelschwerer bis schwerer Psoriasis erhielten entweder $45 \mathrm{mg}$ oder $90 \mathrm{mg}$ Ustekinumab subkutan, zunächst in Woche o und 4, dann alle 12 Wochen. Primärer Endpunkt war der Anteil jener Patienten, deren Hautläsionen sich bis Woche 12 um mindestens 75 Prozent gebessert hatten (PASI 75, Psoriasis Area and Severity Index). Einen solchen Therapieerfolg registrierten die Prüfer mit der niedrigen Dosis in beiden Studien übereinstimmend bei 67 Prozent der Patienten. Mit der hohen Dosis kam es in der ersten Studie bei 66 Prozent der Patienten zu diesem Resultat und in der zweiten bei 76 Prozent. Im Januar 2009 erhielt Ustekinumab die Zulassung für erwachsene Patienten mit mittelschwerer bis schwerer Psoriasis, die auf andere systemische Therapien einschließlich Ciclosporin, Methotrexat und PUVA nicht angesprochen bzw. sie nicht vertragen haben oder bei denen diese Substanzen kontraindiziert sind. ars

Nach Informationen von

Janssen-Cilag
- Stelara ${ }^{\circledR}$ (Ustekinumab) vom Unternehmen Janssen-Cilag ist ein Biological zur Behandlung der mittelschweren bis schweren Plaque-Psoriasis. Es bessert nicht nur die Läsionen der Haut, und zwar effektiver als ein TNF-alpha-Hemmer, sondern auch Manifestationen an Finger- und Zehennägeln. Das erhöht die Lebensqualität der Patienten.

Ustekinumab ermöglicht eine maßgeschneiderte Behandlung bei Psoriasis: Der monoklonale Antikörper ist genau auf die

\section{Galenus-von-Pergamon-Preis 2010}

- Die auf dieser Seite vorgestellten Medikamente Toctino ${ }^{\circledR}$ und Stelara ${ }^{\circledR}$ sind unter den diesjährigen zwölf Bewerbern für den Galenus-von-Pergamon-Preis diejenigen aus dem Bereich Dermatologie. Der Galenus-Preis würdigt herausragende, in Deutschland zugelassene Arzneiinnovationen; er ist mit 10.000
Euro dotiert. Die internationale Stiftergemeinschaft des „Prix Galien“ wird hierzulande von der Springer Medizin Ärzte Zeitung Verlagsgesellschaft vertreten, die auch den nationalen Preis stiftet. Die Ehrung der Preisträger findet am 21. Oktober 2010 in Berlin statt. 